\title{
The universality of quadratic $L$-series for prime discriminants
}

\author{
by \\ Hidehiko Mishou (Nagoya) and Hirofumi Nagoshi (Niigata)
}

1. Introduction and statement of results. For an odd prime $p$ let $\lambda_{p}$ denote the real Dirichlet character modulo $p$ given by the Legendre symbol $(\dot{\bar{p}})$. Let $L(s, \chi)$ be the Dirichlet $L$-function associated with a character $\chi$. The value distribution of $L\left(s, \lambda_{p}\right)$ for a complex number $s$ with $\operatorname{Re} s>1 / 2$ as $p$ varies over the odd primes is investigated e.g. in [El]. The main purpose of the present paper is to study the functional distribution of $L\left(s, \lambda_{p}\right)$ on $D$, as $p$ varies over the primes in an arithmetic progression; here and henceforth $D$ denotes the strip $\{s \in \mathbb{C} \mid 1 / 2<\operatorname{Re} s<1\}$. More precisely, we shall establish the so-called universality theorem for $L\left(s, \lambda_{p}\right)$ in the $p$-aspect.

The universality theorem was first discovered by Voronin ([Vo], $[\mathrm{KV}]$ ) for the Riemann zeta-function $\zeta(s)$ in the $t$-aspect; he showed the following.

TheOrem ([Vo]). Let $0<r<1 / 4$ and $h(s)$ be a continuous function on the disk $|s| \leq r$ which is holomorphic and has no zeros in $|s|<r$. Then for any $\varepsilon>0$ there exists a real number $t$ such that

$$
\max _{|s| \leq r}|\zeta(s+3 / 4+i t)-h(s)|<\varepsilon .
$$

The universality theorem for a Dirichlet $L$-function $L(s, \chi)$ in the $t$ aspect was obtained by Bagchi [B1], [B2], Gonek [Go] and Voronin (see $[\mathrm{KV}$, Chapter VII, Section 3]) independently; indeed, the joint universality theorem was shown.

Furthermore, Bagchi [B1], Eminyan [Em] and Gonek [Go] independently showed an analogous result for Dirichlet $L$-functions in another aspect. In fact, they established the universality theorem for the family of $L(s, \chi)$ 's as $\chi$ varies over the set of characters modulo $q$ with $q$ large.

We denote by $\mathbb{R}, \mathbb{R}^{+}, \mathbb{Z}$ and $\mathbb{N}$ the set of all real numbers, positive real numbers, integers and positive integers, respectively. For a discriminant $d$, let $\chi_{d}$ denote the real Dirichlet character modulo $|d|$ defined by the Kronecker

2000 Mathematics Subject Classification: Primary 11M06, 41A30; Secondary 11R42. 
symbol $\left(\frac{d}{.}\right)_{\mathrm{K}}$. Letting $\gamma$ stand for the plus sign or the minus sign, we define $\mathcal{D}^{\gamma}:= \begin{cases}\{d>0 \mid d \text { is a square-free integer, } d \equiv 1 \bmod 8, d \neq 1\} & \text { if } \gamma \text { is }+, \\ \{d<0 \mid d \text { is a square-free integer, } d \equiv 1 \bmod 8\} & \text { if } \gamma \text { is }-,\end{cases}$ and

$$
\mathcal{D}_{X}^{\gamma}:=\left\{d \in \mathcal{D}^{\gamma}|| d \mid \leq X\right\} \quad \text { for } X \in \mathbb{R}^{+} .
$$

The authors [MN1] have recently obtained the following universality theorem, which is an analogue of Bagchi, Eminyan and Gonek's result above for the family $\left\{L\left(s, \chi_{d}\right) \mid d \in \mathcal{D}^{\gamma}\right\}$ of $L$-functions associated with real characters $\chi_{d}$ : Let $\Omega, h(s)$ and $K$ be as in Theorem 1.1 below. Then for any $\varepsilon>0$ we have

$$
\liminf _{X \rightarrow \infty} \frac{1}{\# \mathcal{D}_{X}^{\gamma}} \#\left\{d \in \mathcal{D}_{X}^{\gamma}\left|\max _{s \in K}\right| L\left(s, \chi_{d}\right)-h(s) \mid<\varepsilon\right\}>0 .
$$

In the present paper we investigate the universality theorem for $L\left(s, \lambda_{p}\right)$ in the prime $p$-aspect, as mentioned above. Noting that $L\left(s, \lambda_{p}\right)$ is equal to $L\left(s, \chi_{q}\right)$ with a certain integer $q$ (see (3.3)), we will deal with $L\left(s, \chi_{q}\right)$ instead of $L\left(s, \lambda_{p}\right)$. Throughout let $\gamma \in\{+,-\}$ and let $m$ and $a=a(\gamma)$ be any fixed positive integers such that $\operatorname{gcd}(m, a)=1,8 \mid m, a \equiv 1 \bmod 4$ if $\gamma$ is + and $a \equiv 3 \bmod 4$ if $\gamma$ is - . We define

$$
\mathcal{P}^{\gamma}(m, a):= \begin{cases}\{p \mid p \text { is a prime, } p \equiv a \bmod m\} & \text { if } \gamma \text { is }+, \\ \{-p \mid p \text { is a prime, } p \equiv a \bmod m\} & \text { if } \gamma \text { is }-,\end{cases}
$$

and

$$
\mathcal{P}_{X}^{\gamma}(m, a):=\left\{q \in \mathcal{P}^{\gamma}(m, a)|| q \mid \leq X\right\} \quad \text { for } X>0 .
$$

By the prime number theorem for arithmetic progressions,

$$
\# \mathcal{P}_{X}^{\gamma}(m, a) \sim \frac{1}{\varphi(m)} \frac{X}{\log X} \quad \text { as } X \rightarrow \infty,
$$

where $\varphi(m)$ denotes the Euler totient function. Every integer $q$ in $\mathcal{P}^{\gamma}(m, a)$ is a prime discriminant (for its definition, see e.g. [Ay, p. 310], [Da, p. 41]). In the following, the letter $p$ will stand for a prime number and $q$ for a prime discriminant.

TheOREM 1.1. Let $\gamma \in\{+,-\}$. Let $m, a \in \mathbb{N}$ be as above. Let $\Omega$ be a simply connected region in $D$ which is symmetric with respect to the real axis. Suppose that $h(s)$ is a holomorphic function on $\Omega$ which has no zeros on $\Omega$ and is $\mathbb{R}^{+}$-valued on the set $\Omega \cap \mathbb{R}$. Let $K$ be a compact set in $\Omega$, and $\varepsilon>0$. Then there exist infinitely many $q \in \mathcal{P}^{\gamma}(m, a)$ such that $\max _{s \in K} \mid L\left(s, \chi_{q}\right)-$ $h(s) \mid<\varepsilon$. More precisely, we have

$$
\liminf _{X \rightarrow \infty} \frac{1}{\# \mathcal{P}_{X}^{\gamma}(m, a)} \#\left\{q \in \mathcal{P}_{X}^{\gamma}(m, a)\left|\max _{s \in K}\right| L\left(s, \chi_{q}\right)-h(s) \mid<\varepsilon\right\}>0 .
$$


It should be noted that the results (1.1) and (1.3) do not directly imply each other, because the density of the set $\mathcal{P}^{\gamma}(m, a)$ in $\mathcal{D}^{\gamma}$ is 0 in the sense that $\# \mathcal{P}_{X}^{\gamma}(m, a) / \# \mathcal{D}_{X}^{\gamma} \rightarrow 0$ as $X \rightarrow \infty$ (see [MN1, Lemma 4.1] and (1.2)).

In the same way as in the present paper, we can generalize (1.1) to the result in which $d$ varies over the fundamental discriminants in the arithmetic progression $\{k m+a \mid k \in \mathbb{Z}\}$, where $m, a \in \mathbb{N}$ are as in Theorem 1.1.

Theorem 1.1 yields the following corollaries, for example. First we get a denseness result on values of $L\left(s, \chi_{q}\right)$ 's for fixed $s \in D$ and variable $q \in$ $\mathcal{P}^{\gamma}(m, a)$. This is analogous to Bohr-Courant's result $[\mathrm{BC}]$ on values of the Riemann zeta-function $\zeta(s)$.

Corollary 1.2 .

(1) Let any $s_{0} \in D$ with $\operatorname{Im} s_{0} \neq 0$ be fixed. Then the set $\left\{L\left(s_{0}, \chi_{q}\right) \mid q \in\right.$ $\left.\mathcal{P}^{\gamma}(m, a)\right\}$ is dense in $\mathbb{C}$. More precisely, for any $z_{0} \in \mathbb{C}$ and $\varepsilon>0$ we have

$$
\liminf _{X \rightarrow \infty} \frac{1}{\# \mathcal{P}_{X}^{\gamma}(m, a)} \#\left\{q \in \mathcal{P}_{X}^{\gamma}(m, a)|| L\left(s_{0}, \chi_{q}\right)-z_{0} \mid<\varepsilon\right\}>0 .
$$

(2) Let $1 / 2<\sigma_{0}<1$ be fixed. Then the set $\left\{L\left(\sigma_{0}, \chi_{q}\right) \mid q \in \mathcal{P}^{\gamma}(m, a)\right\}$ is dense in $\mathbb{R}^{+}$. More precisely, for any $x_{0} \in \mathbb{R}^{+}$and $\varepsilon>0$ we have

$$
\liminf _{X \rightarrow \infty} \frac{1}{\# \mathcal{P}_{X}^{\gamma}(m, a)} \#\left\{q \in \mathcal{P}_{X}^{\gamma}(m, a)|| L\left(\sigma_{0}, \chi_{q}\right)-x_{0} \mid<\varepsilon\right\}>0 .
$$

Next we have a non-vanishing result for $L\left(s, \chi_{q}\right)$ 's on $D$, and the following stronger result.

Corollary 1.3. Let $\alpha, \beta$ be any positive real numbers with $\alpha<\beta$. Let $K$ be a compact set in $D$. Then

$$
\begin{array}{r}
\liminf _{X \rightarrow \infty} \frac{1}{\# \mathcal{P}_{X}^{\gamma}(m, a)} \#\left\{q \in \mathcal{P}_{X}^{\gamma}(m, a)|\alpha<| L\left(s, \chi_{q}\right) \mid<\beta\right. \\
\text { uniformly for } s \in K\}>0 .
\end{array}
$$

Noting that $L\left(s, \chi_{q}\right)$ is $\mathbb{R}$-valued on the real segment $(1 / 2,1)$, we can obtain a result concerning the horizontal distribution of zeros of the derivatives $L^{(r)}\left(s, \chi_{q}\right)$ on $(1 / 2,1)$ in the $q$-aspect.

Corollary 1.4. Let $\alpha, \beta \in \mathbb{R}$ with $1 / 2<\alpha<\beta<1$ and $r^{\prime}, N \in \mathbb{N}$. Then there exist infinitely many $q \in \mathcal{P}^{\gamma}(m, a)$ such that for every integer $r$ with $1 \leq r \leq r^{\prime}$ the rth derivative $L^{(r)}\left(s, \chi_{q}\right)$ has at least $N$ zeros on the interval $[\alpha, \beta] \subset \mathbb{R}$. More precisely,

$$
\begin{array}{r}
\liminf _{X \rightarrow \infty} \frac{1}{\# \mathcal{P}_{X}^{\gamma}(m, a)} \#\left\{q \in \mathcal{P}_{X}^{\gamma}(m, a) \mid L^{(r)}\left(s, \chi_{q}\right) \text { has at least } N\right. \text { zeros } \\
\text { on } \left.[\alpha, \beta] \text { for every } r=1, \ldots, r^{\prime}\right\}>0 .
\end{array}
$$


We shall also study the denseness result on values of $L\left(s, \chi_{q}\right)$ for a fixed complex number $s \neq 1$ with $\operatorname{Re} s=1$ and variable $q \in \mathcal{P}^{\gamma}(m, a)$.

Theorem 1.5. Let $t \in \mathbb{R}-\{0\}$ be fixed. Then the set $\left\{L\left(1+i t, \chi_{q}\right) \mid q \in\right.$ $\left.\mathcal{P}^{\gamma}(m, a)\right\}$ is dense in $\mathbb{C}$. More precisely, for any $z_{0} \in \mathbb{C}$ and $\varepsilon>0$ we have

$$
\liminf _{X \rightarrow \infty} \frac{1}{\# \mathcal{P}_{X}^{\gamma}(m, a)} \#\left\{q \in \mathcal{P}_{X}^{\gamma}(m, a)|| L\left(1+i t, \chi_{q}\right)-z_{0} \mid<\varepsilon\right\}>0 .
$$

In [MN2] the authors showed an analogue of Theorem 1.5 for $L\left(1, \lambda_{p}\right)$ and deduced from it a quantitative result for a problem of Ayoub-ChowlaWalum on certain character sums.

2. Denseness lemma. The purpose of this section is to show Proposition 2.3 below. For $s \in \mathbb{C}$ we write $s=\sigma+i t$ with $\sigma, t \in \mathbb{R}$. The next lemma is proved in [MN1, Proposition 2.4].

Lemma 2.1. Let $\Omega$ be a simply connected region in $D$ symmetric with respect to the real axis, as in Theorem 1.1. Let $U$ be a bounded, simply connected region in $\Omega$ which is symmetric with respect to the real axis and which satisfies $\bar{U} \subset \Omega$, where $\bar{U}$ denotes the closure of $U$. Suppose that $g(s)$ is a holomorphic function on $\Omega$ which is $\mathbb{R}$-valued on the interval $\Omega \cap \mathbb{R}$. Let $y>0$ be fixed. Then for any $\varepsilon>0$ there exist $\nu \in \mathbb{R}^{+}$and $c_{p} \in\{1,-1\}$, for each prime $p$ with $y \leq p \leq \nu$, such that

$$
\int_{U}\left|g(s)-\sum_{y \leq p \leq \nu} \frac{c_{p}}{p^{s}}\right|^{2} d \sigma d t<\varepsilon .
$$

The next lemma is a generalization of $[\mathrm{Ti}$, p. 303, Lemma] and was obtained in [MN1, Lemma 2.5].

Lemma 2.2. Let $U$ be a bounded region in $\mathbb{C}$. Let $K$ be a compact subset of $\mathbb{C}$ such that $K \subset U$. Let $B>0$. Suppose that $f(s)$ is a holomorphic function on $U$ satisfying $\int_{U}|f(s)|^{2} d \sigma d t \leq B$. Then $\max _{s \in K}|f(s)| \leq$ $b(U, K) B^{1 / 2}$, where $b(U, K)$ is a certain positive constant depending only on $U$ and $K$.

Proposition 2.3. Let $\Omega$ be a simply connected region in $D$ symmetric with respect to the real axis. Suppose that $g(s)$ is a holomorphic function on $\Omega$ which is $\mathbb{R}$-valued on $\Omega \cap \mathbb{R}$. Let $K$ be a compact set in $\Omega$ and $\nu_{1} \in \mathbb{R}^{+}$ with $\nu_{1}>m+1$. Let $a_{p} \in\{1,-1\}$ for each prime $p$ with $p \mid m$. Then for any $\varepsilon>0$ there exist $\nu>\nu_{1}$ and $a_{p} \in\{1,-1\}$, for each prime $p$ with $p \leq \nu$ and $p \nmid m$, such that

$$
\max _{s \in K}\left|g(s)-\log \prod_{p \leq \nu}\left(1-\frac{a_{p}}{p^{s}}\right)^{-1}\right|<\varepsilon,
$$


where

$$
\log \prod_{p \leq \nu}\left(1-\frac{a_{p}}{p^{s}}\right)^{-1}=-\sum_{p \leq \nu} \log \left(1-\frac{a_{p}}{p^{s}}\right)=\sum_{p \leq \nu} \sum_{n=1}^{\infty} \frac{a_{p}^{n}}{n p^{n s}} .
$$

Proof. Take a bounded, simply connected region $U$ in $\Omega$ which is symmetric with respect to the real axis and which satisfies $K \subset U$ and $\bar{U} \subset \Omega$. Set $\sigma_{1}:=\min \{\operatorname{Re} s \mid s \in \bar{U}\}>1 / 2$. Let $\varepsilon>0$ be arbitrary. Fix a real number $y$ satisfying $y>\nu_{1}$ and $y^{1-2 \sigma_{1}} /\left(2 \sigma_{1}-1\right)<\varepsilon$. Then we have

$$
\begin{aligned}
\sum_{p \geq y} \sum_{n=2}^{\infty} \frac{1}{n p^{n \sigma_{1}}} & \leq \sum_{p \geq y} \sum_{n=2}^{\infty} \frac{1}{p^{n \sigma_{1}}}=\sum_{p \geq y} \frac{p^{-2 \sigma_{1}}}{1-p^{-\sigma_{1}}} \\
& \ll \sum_{n \geq y, n \in \mathbb{N}} \frac{1}{n^{2 \sigma_{1}}} \ll \frac{y^{1-2 \sigma_{1}}}{2 \sigma_{1}-1}<\varepsilon .
\end{aligned}
$$

Set $a_{p}=1$ for each prime $p$ with $p<y$ and $p \nmid m$. From Lemma 2.1 it follows that there exist $\nu \geq y$ and $c_{p} \in\{1,-1\}$, for each prime $p$ with $y \leq p \leq \nu$, such that

$$
\int_{U}\left|\left(g(s)-\sum_{p<y} \sum_{n=1}^{\infty} \frac{a_{p}^{n}}{n p^{n s}}\right)-\sum_{y \leq p \leq \nu} \frac{c_{p}}{p^{s}}\right|^{2} d \sigma d t<\varepsilon^{2} .
$$

This and Lemma 2.2 yield

$$
\max _{s \in K}\left|g(s)-\sum_{p<y} \sum_{n=1}^{\infty} \frac{a_{p}^{n}}{n p^{n s}}-\sum_{y \leq p \leq \nu} \frac{c_{p}}{p^{s}}\right| \ll_{U, K} \varepsilon .
$$

For each prime $p$ with $y \leq p \leq \nu$ we set $a_{p}=c_{p}$. Then we obtain, by (2.1) and (2.2),

$$
\begin{aligned}
& \max _{s \in K}\left|g(s)-\log \prod_{p \leq \nu}\left(1-\frac{a_{p}}{p^{s}}\right)^{-1}\right| \\
&=\max _{s \in K}\left|g(s)-\sum_{p<y} \sum_{n=1}^{\infty} \frac{a_{p}^{n}}{n p^{n s}}-\sum_{y \leq p \leq \nu} \frac{c_{p}}{p^{s}}-\sum_{y \leq p \leq \nu} \sum_{n=2}^{\infty} \frac{c_{p}^{n}}{n p^{n s}}\right| \\
& \quad \leq \max _{s \in K}\left|g(s)-\sum_{p<y} \sum_{n=1}^{\infty} \frac{a_{p}^{n}}{n p^{n s}}-\sum_{y \leq p \leq \nu} \frac{c_{p}}{p^{s}}\right|+\max _{s \in K}\left|\sum_{y \leq p \leq \nu} \sum_{n=2}^{\infty} \frac{c_{p}^{n}}{n p^{n s}}\right| \\
& \ll_{U, K} \varepsilon+\sum_{p \geq y} \sum_{n=2}^{\infty} \frac{1}{n p^{n \sigma_{1}}} \ll \varepsilon,
\end{aligned}
$$

which completes the proof. 
3. Approximation by finite Euler products. As usual, let $\pi(X)$ denote the number of primes not exceeding $X \in \mathbb{R}^{+}$. For large $X \in \mathbb{R}^{+}$, let $R_{X}$ denote the set

$$
\left\{s=\sigma+i t \in \mathbb{C}\left|1 / 2+(\log \log \log X)^{-1 / 2} \leq \sigma \leq 5 / 4,\right| t \mid<X^{\frac{1}{13}(2 \sigma-1)}\right\}
$$

and put

$$
h_{X}:=(\log \log X)^{2} .
$$

The next lemma is obtained in [El, Lemma 8].

Lemma 3.1. For all large $X$ and uniformly for $s \in R_{X}$ we have

$$
\sum_{\substack{3 \leq r \leq X \\ r: \text { prime }}}\left|L\left(s, \lambda_{r}\right)-\prod_{p \leq h_{X}}\left(1-\frac{\lambda_{r}(p)}{p^{s}}\right)^{-1}\right|^{2} \ll \pi(X) h_{X}^{1-2 \sigma}\left(\log h_{X}\right)^{3}(2 \sigma-1)^{-4} .
$$

Recall that for an odd prime $r$ and a positive integer $n$ we have the relation (see e.g. [Ay, p. 290, Lemma 2.2])

$$
\left(\frac{n}{r}\right)= \begin{cases}\left(\frac{r}{n}\right)_{\mathrm{K}} & \text { if } r \equiv 1 \bmod 4, \\ \left(\frac{-r}{n}\right)_{\mathrm{K}} & \text { if } r \equiv 3 \bmod 4\end{cases}
$$

and hence

$$
L\left(s, \lambda_{r}\right)= \begin{cases}L\left(s, \chi_{r}\right) & \text { if } r \equiv 1 \bmod 4, \\ L\left(s, \chi_{-r}\right) & \text { if } r \equiv 3 \bmod 4 .\end{cases}
$$

Proposition 3.2. Let $\varepsilon>0$ and $K$ be a compact set in the region $1 / 2<\operatorname{Re} s<5 / 4$. Define $\mathcal{A}_{X}^{\gamma}(m, a)=\mathcal{A}_{X}^{\gamma}(m, a, \varepsilon, K)$ by

$$
\mathcal{A}_{X}^{\gamma}(m, a):=\left\{q \in \mathcal{P}_{X}^{\gamma}(m, a)\left|\max _{s \in K}\right| L\left(s, \chi_{q}\right)-\prod_{p \leq h_{X}}\left(1-\frac{\chi_{q}(p)}{p^{s}}\right)^{-1} \mid<\varepsilon\right\} .
$$

Then

$$
\frac{\# \mathcal{A}_{X}^{\gamma}(m, a)}{\# \mathcal{P}_{X}^{\gamma}(m, a)}>1-\varepsilon
$$

if $X$ is sufficiently large.

Proof. Take an open rectangle $U$ of the form $\left\{s \in \mathbb{C} \mid \sigma_{1}<\operatorname{Re} s<\sigma_{2}\right.$, $|\operatorname{Im} s|<A\}$ satisfying $1 / 2<\sigma_{1}<\min \{\operatorname{Re} s \mid s \in K\} \leq \max \{\operatorname{Re} s \mid s \in K\}$ $<\sigma_{2}<5 / 4$ and $\max \{|\operatorname{Im} s| \mid s \in K\}<A$. Then $K \subset U$. For large $X \in \mathbb{R}^{+}$ we define $\widetilde{\mathcal{A}}_{X}^{\gamma}(m, a)$ to be the set

$$
\left\{q \in \mathcal{P}_{X}^{\gamma}(m, a)\left|\int_{U}\right| L\left(s, \chi_{q}\right)-\left.\prod_{p \leq h_{X}}\left(1-\frac{\chi_{q}(p)}{p^{s}}\right)^{-1}\right|^{2} d \sigma d t<\frac{\varepsilon^{2}}{b(U, K)^{2}}\right\}
$$


where $b(U, K)$ is the constant in Lemma 2.2. By Lemma 2.2,

$$
\widetilde{\mathcal{A}}_{X}^{\gamma}(m, a) \subset \mathcal{A}_{X}^{\gamma}(m, a) .
$$

From Lemma 3.1, (3.2), (3.3), the prime number theorem, and (1.2), we infer that for all large $X$,

$$
\begin{aligned}
\sum_{q \in \mathcal{P}_{X}^{\gamma}(m, a)} \int_{U}\left|L\left(s, \chi_{q}\right)-\prod_{p \leq h_{X}}\left(1-\frac{\chi_{q}(p)}{p^{s}}\right)^{-1}\right|^{2} d \sigma d t \\
\quad \leq \sum_{\substack{3 \leq r \leq X \\
r: \text { prime }}} \int_{U}\left|L\left(s, \lambda_{r}\right)-\prod_{p \leq h_{X}}\left(1-\frac{\lambda_{r}(p)}{p^{s}}\right)^{-1}\right|^{2} d \sigma d t \\
\ll_{U} \pi(X) h_{X}^{1-2 \sigma_{1}}\left(\log h_{X}\right)^{3}\left(2 \sigma_{1}-1\right)^{-4} \\
\ll \varphi(m) \# \mathcal{P}_{X}^{\gamma}(m, a) h_{X}^{1-2 \sigma_{1}}\left(\log h_{X}\right)^{3}\left(2 \sigma_{1}-1\right)^{-4} .
\end{aligned}
$$

Since $h_{X}^{1-2 \sigma_{1}}\left(\log h_{X}\right)^{3} \rightarrow 0$ as $X \rightarrow \infty$, it follows from (3.6) that there exists a large number $X_{0}=X_{0}(\varepsilon, U, K, m)$ such that for all $X>X_{0}$,

$$
\begin{aligned}
\sum_{q \in \mathcal{P}_{X}^{\gamma}(m, a)} \int_{U}\left|L\left(s, \chi_{q}\right)-\prod_{p \leq h_{X}}\left(1-\frac{\chi_{q}(p)}{p^{s}}\right)^{-1}\right|^{2} d \sigma d t & \\
& <\frac{\varepsilon^{3}}{b(U, K)^{2}} \# \mathcal{P}_{X}^{\gamma}(m, a) .
\end{aligned}
$$

Now assume that there exists a real number $X>X_{0}$ such that $\#\left(\mathcal{P}_{X}^{\gamma}(m, a)\right.$ $\left.-\widetilde{\mathcal{A}}_{X}^{\gamma}(m, a)\right) \geq \varepsilon \# \mathcal{P}_{X}^{\gamma}(m, a)$. For this $X$ we have, by $(3.4)$,

$$
\begin{gathered}
\sum_{q \in \mathcal{P}_{X}^{\gamma}(m, a)} \int_{U}\left|L\left(s, \chi_{q}\right)-\prod_{p \leq h_{X}}\left(1-\frac{\chi_{q}(p)}{p^{s}}\right)^{-1}\right|^{2} d \sigma d t \\
\geq \sum_{q \in \mathcal{P}_{X}^{\gamma}(m, a)-\tilde{\mathcal{A}}_{X}^{\gamma}(m, a)} \int_{U}\left|L\left(s, \chi_{q}\right)-\prod_{p \leq h_{X}}\left(1-\frac{\chi_{q}(p)}{p^{s}}\right)^{-1}\right|^{2} d \sigma d t \\
\geq \varepsilon \# \mathcal{P}_{X}^{\gamma}(m, a) \frac{\varepsilon^{2}}{b(U, K)^{2}}=\frac{\varepsilon^{3}}{b(U, K)^{2}} \# \mathcal{P}_{X}^{\gamma}(m, a) .
\end{gathered}
$$

However, this contradicts (3.7). Hence for any $X>X_{0}$ we have

$$
\#\left(\mathcal{P}_{X}^{\gamma}(m, a)-\widetilde{\mathcal{A}}_{X}^{\gamma}(m, a)\right)<\varepsilon \# \mathcal{P}_{X}^{\gamma}(m, a)
$$

that is, $\# \widetilde{\mathcal{A}}_{X}^{\gamma}(m, a) / \# \mathcal{P}_{X}^{\gamma}(m, a)>1-\varepsilon$. This and (3.5) complete the proof.

4. Results on characters $\chi_{q}$ for prime discriminants $q$. The aim of this section is to obtain Proposition 4.3. As before, the letter $\gamma$ denotes the plus sign or the minus sign. For $X \in \mathbb{R}^{+}$we define $I_{X}$ to be the interval 
$[0, X]$ if $\gamma$ is + , and $[-X, 0]$ if $\gamma$ is - . We define

$$
\delta=\delta(\gamma)= \begin{cases}1 & \text { if } \gamma \text { is }+, \\ -1 & \text { if } \gamma \text { is }-.\end{cases}
$$

Lemma 4.1. Fix a number $\nu \in \mathbb{R}^{+}$such that $\pi(\nu)>\pi(m)$. Let $a_{p} \in$ $\{1,-1\}$ for each prime $p$ satisfying $p \leq \nu$ and $p \nmid m$. Define $\mathcal{P}_{X, \nu}^{\gamma}(m, a)=$ $\mathcal{P}_{X, \nu}^{\gamma}\left(m, a,\left\{a_{p}\right\}\right)$ to be the set

$$
\left\{q \in \mathcal{P}_{X}^{\gamma}(m, a) \mid \chi_{q}(p)=a_{p} \text { for every prime } p \text { with } p \leq \nu \text { and } p \nmid m\right\},
$$

and put $C_{\nu}(m):=\prod_{p \leq \nu, p \nmid m} \frac{1}{2}$. Then

$$
\lim _{X \rightarrow \infty} \frac{\# \mathcal{P}_{X, \nu}^{\gamma}(m, a)}{\# \mathcal{P}_{X}^{\gamma}(m, a)}=C_{\nu}(m) .
$$

Proof. In general, for $n \in \mathbb{N}$ and $b \in \mathbb{Z}$, we denote by $[b]_{n}$ the set of all integers $x$ such that $x \equiv b \bmod n$, that is, the residue class mod $n$ which $b$ belongs to.

Let $p$ be an odd prime. Let $\mathcal{Q}_{p}$ be the set of all residue classes $[b]_{p} \bmod p$ such that $b$ is a quadratic residue $\bmod p$, other than the residue class $[0]_{p}$, and let $\mathcal{Q}_{p}^{\prime}$ be the set of all residue classes $[c]_{p} \bmod p$ such that $c$ is a quadratic non-residue mod $p$. It is well known that

$$
\# \mathcal{Q}_{p}=\# \mathcal{Q}_{p}^{\prime}=\frac{p-1}{2} .
$$

In view of the definitions of Kronecker's symbol and Legendre's symbol, a discriminant $q$ satisfies $\chi_{q}(p)=a_{p}$ if and only if $q$ belongs to one of residue classes in $\mathcal{Q}_{p}$ if $a_{p}=1$ and in $\mathcal{Q}_{p}^{\prime}$ if $a_{p}=-1$. From this, (4.2) and the Chinese remainder theorem, it follows, for an integer $r$ such that $\delta r$ is a prime number, that $r$ satisfies $r \equiv \delta a \bmod m$ (i.e. $r \in \mathcal{P}^{\gamma}(m, a)$ ) and $\chi_{r}(p)=a_{p}$ for every prime $p$ with $p \leq \nu$ and $p \nmid m$ if and only if $r$ belongs to one of exactly $\prod_{p \leq \nu, p \nmid m}(p-1) / 2$ distinct residue classes $\bmod Q$, where

$$
Q=Q(m, \nu):=m \prod_{p \leq \nu, p \nmid m} p
$$

and $\delta$ is as in (4.1). Let $\mathcal{R}^{\gamma}=\mathcal{R}^{\gamma}(m, a, \nu)$ denote the set of those residue classes $\bmod Q$, so that

$$
\# \mathcal{R}^{\gamma}=\prod_{p \leq \nu, p \nmid m} \frac{p-1}{2} .
$$


Thus

(4.4) $\mathcal{P}_{X, \nu}^{\gamma}(m, a)=\left\{r \in I_{X} \mid \delta r\right.$ is a prime, $r \equiv \delta a \bmod m$,

$$
\begin{aligned}
& \left.\chi_{r}(p)=a_{p} \text { for every prime } p \text { with } p \leq \nu \text { and } p \nmid m\right\} \\
= & \bigcup_{[c]_{Q} \in \mathcal{R}^{\gamma}}\left\{r \in I_{X} \mid \delta r \text { is a prime, } r \equiv c \bmod Q\right\} .
\end{aligned}
$$

We note that if $[c]_{Q} \in \mathcal{R}^{\gamma}$ then

$$
\operatorname{gcd}(c, Q)=1,
$$

since $[0]_{p} \notin \mathcal{Q}_{p}$ and $[0]_{p} \notin \mathcal{Q}_{p}^{\prime}$ for all primes $p$ with $p \leq \nu$ and $p \nmid m$, and $\operatorname{gcd}(a, m)=1$.

From (4.4) we have

$$
\# \mathcal{P}_{X, \nu}^{\gamma}(m, a)=\sum_{[c]_{Q} \in \mathcal{R}^{\gamma}} \sum_{\substack{r \in I_{X}, \delta r: \text { prime } \\ r \equiv c \bmod Q}} 1=\sum_{[c]_{Q} \in \mathcal{R}^{\gamma}} \sum_{\substack{p \leq X \\ p \equiv \delta c \bmod Q}} 1 .
$$

By the prime number theorem for arithmetic progressions and (4.5),

$$
\sum_{\substack{p \leq X \\ p \equiv \delta c \bmod Q}} 1 \sim \frac{1}{\varphi(Q)} \frac{X}{\log X} \quad \text { as } X \rightarrow \infty .
$$

Note that the right-hand side of (4.6) is independent of $[c]_{Q} \in \mathcal{R}^{\gamma}$. Therefore for fixed $\nu$ we have

$$
\begin{aligned}
\# \mathcal{P}_{X, \nu}^{\gamma}(m, a) & \sim \frac{\# \mathcal{R}^{\gamma}}{\varphi(Q)} \frac{X}{\log X} \\
& =\left(\prod_{p \leq \nu, p \nmid m} \frac{1}{2}\right) \frac{X}{\varphi(m) \log X}=\frac{C_{\nu}(m)}{\varphi(m)} \frac{X}{\log X}
\end{aligned}
$$

as $X \rightarrow \infty$, using (4.3) and the fact

$$
\varphi(Q)=\varphi(m) \prod_{p \leq \nu, p \nmid m} \varphi(p)=\varphi(m) \prod_{p \leq \nu, p \nmid m}(p-1) .
$$

Thus (4.7) and (1.2) give us

$$
\frac{\# \mathcal{P}_{X, \nu}^{\gamma}(m, a)}{\# \mathcal{P}_{X}^{\gamma}(m, a)}=\frac{\# \mathcal{P}_{X, \nu}^{\gamma}(m, a)}{\frac{C_{\nu}(m)}{\varphi(m)} \frac{X}{\log X}} \frac{\frac{C_{\nu}(m)}{\varphi(m)} \frac{X}{\log X}}{\frac{1}{\varphi(m)} \frac{X}{\log X}} \frac{\frac{1}{\varphi(m)} \frac{X}{\log X}}{\# \mathcal{P}_{X}^{\gamma}(m, a)} \rightarrow C_{\nu}(m)
$$

as $X \rightarrow \infty$. This completes the proof.

Lemma 4.2. Fix $\nu \in \mathbb{R}^{+}$such that $\pi(\nu)>\pi(m)$. Let $a_{p} \in\{1,-1\}$ for each prime $p$ with $p \leq \nu$ and $p \nmid m$. Let $\mathcal{P}_{X, \nu}^{\gamma}(m, a)$ and $C_{\nu}(m)$ be as in Lemma $4.1, h_{X}=(\log \log X)^{2}$ be as in $(3.1)$, and $\sigma_{1}>1 / 2$. Then for all 
large $X$ and uniformly for $s \in \mathbb{C}$ with $\operatorname{Re} s \geq \sigma_{1}$ we have

$$
\sum_{q \in \mathcal{P}_{X, \nu}^{\gamma}(m, a)}\left|\sum_{\nu<p \leq h_{X}} \frac{\chi_{q}(p)}{p^{s}}\right|^{2} \ll \frac{\nu^{1-2 \sigma_{1}}}{2 \sigma_{1}-1} C_{\nu}(m) \# \mathcal{P}_{X}^{\gamma}(m, a) .
$$

Proof. Let $Q$ and $\mathcal{R}^{\gamma}$ be as in the proof of Lemma 4.1. From (4.4) it follows that

$$
\begin{aligned}
\sum_{q \in \mathcal{P}_{X, \nu}^{\gamma}(m, a)}\left|\sum_{\nu<p \leq h_{X}} \frac{\chi_{q}(p)}{p^{s}}\right|^{2} & =\sum_{[c]_{Q} \in \mathcal{R}^{\gamma}} \sum_{\substack{r \in I_{X}, \delta r: \operatorname{prime} \\
r \equiv c \bmod Q}}\left|\sum_{\nu<p \leq h_{X}} \frac{\chi_{r}(p)}{p^{s}}\right|^{2} \\
& =\sum_{[c]_{Q} \in \mathcal{R}^{\gamma}} \sum_{\substack{u \leq X, u: \operatorname{prime} \\
u \equiv \delta c \bmod Q}}\left|\sum_{\nu<p \leq h_{X}} \frac{\chi_{\delta u}(p)}{p^{s}}\right|^{2} .
\end{aligned}
$$

For $[c]_{Q} \in \mathcal{R}^{\gamma}$ we have

$$
\begin{aligned}
& \sum_{\substack{u \leq X, u: \operatorname{prime} \\
u \equiv \delta c \bmod Q}}\left|\sum_{\nu<p \leq h_{X}} \frac{\chi_{\delta u}(p)}{p^{s}}\right|^{2} \\
& =\sum_{\substack{u \leq X, u: \text { prime } \\
u \equiv \delta c \bmod Q}}\left(\sum_{\nu<p \leq h_{X}} \frac{\left|\chi_{\delta u}(p)\right|^{2}}{\left|p^{s}\right|^{2}}+\sum_{\substack{p_{1}, p_{2}: \text { primes, } p_{1} \neq p_{2} \\
\nu<p_{1}, p_{2} \leq h_{X}}} \frac{\chi_{\delta u}\left(p_{1}\right) \overline{\chi_{\delta u}\left(p_{2}\right)}}{p_{1}^{s} \overline{p_{2}^{s}}}\right) \\
& =\sum_{\nu<p \leq h_{X}} \frac{1}{\left|p^{s}\right|^{2}} \sum_{\substack{u \leq X, u: \operatorname{prime} \\
u \equiv \delta c \bmod Q}}\left|\chi_{\delta u}(p)\right|^{2} \\
& +\sum_{\substack{p_{1}, p_{2}: \text { primes, } p_{1} \neq p_{2} \\
\nu<p_{1}, p_{2} \leq h_{X}}} \frac{1}{p_{1}^{s} \overline{\overline{p_{2}^{s}}}} \sum_{\substack{u \leq X, u: \text { prime } \\
u \equiv \delta c \bmod Q}} \chi_{\delta u}\left(p_{1}\right) \overline{\chi_{\delta u}\left(p_{2}\right)} \\
& =S_{1}+S_{2}, \quad \text { say. }
\end{aligned}
$$

Using the prime number theorem for arithmetic progressions, we deduce that for all $s \in \mathbb{C}$ with $\operatorname{Re} s \geq \sigma_{1}$

$$
\begin{aligned}
\left|S_{1}\right| & \leq \sum_{\nu<p \leq h_{X}} \frac{1}{p^{2 \sigma_{1}}} \sum_{\substack{u \leq X, u: \text { prime } \\
u \equiv \delta c \bmod Q}} 1 \\
& \ll\left(\sum_{n>\nu, n \in \mathbb{N}} \frac{1}{n^{2 \sigma_{1}}}\right) \frac{1}{\varphi(Q)} \frac{X}{\log X} \\
& \ll \frac{\nu^{1-2 \sigma_{1}}}{2 \sigma_{1}-1} \frac{1}{\varphi(Q)} \frac{X}{\log X} .
\end{aligned}
$$


Next we shall consider the sum $S_{2}$. Fix two distinct primes $p_{1}, p_{2}$ satisfying $\nu<p_{1} \leq h_{X}$ and $\nu<p_{2} \leq h_{X}$. Then by the definition of the Kronecker symbol and the orthogonality relation for Dirichlet characters, we have

$$
\begin{aligned}
& \sum_{\substack{u \leq X, u: \operatorname{prime} \\
u \equiv \delta c \bmod Q}} \chi_{\delta u}\left(p_{1}\right) \overline{\chi_{\delta u}\left(p_{2}\right)}=\sum_{\substack{u \leq X, u: \operatorname{prime} \\
u \equiv \delta c \bmod Q}}\left(\frac{\delta u}{p_{1}}\right)\left(\frac{\delta u}{p_{2}}\right) \\
& =\sum_{u \leq X, u: \operatorname{prime}}\left(\frac{\delta u}{p_{1}}\right)\left(\frac{\delta u}{p_{2}}\right) \frac{1}{\varphi(Q)} \sum_{\lambda \bmod Q} \lambda(u) \overline{\lambda(\delta c)} \\
& =\frac{1}{\varphi(Q)}\left(\frac{\delta}{p_{1}}\right)\left(\frac{\delta}{p_{2}}\right) \sum_{\lambda \bmod Q} \overline{\lambda(\delta c)} \sum_{u \leq X, u: \operatorname{prime}}\left(\frac{u}{p_{1}}\right)\left(\frac{u}{p_{2}}\right) \lambda(u),
\end{aligned}
$$

where $\sum_{\lambda \text { mod } Q}$ means the sum over all the Dirichlet characters $\lambda \bmod Q$. Since $p_{1}, p_{2}$ and $Q$ are relatively prime in pairs, we find from the Chinese remainder theorem that for any character $\lambda \bmod Q$ the product $\left(\dot{\overline{p_{1}}}\right)\left(\dot{\overline{p_{2}}}\right) \lambda(\cdot)$ is a non-principal Dirichlet character $\bmod p_{1} p_{2} Q$. From this, the SiegelWalfisz theorem (see [Da, p. 132, (3)]) and partial summation, it follows, for fixed $\nu$, that for all large $X$ and all pairs of distinct primes $\left(p_{1}, p_{2}\right)$ satisfying $\nu<p_{1} \leq h_{X}$ and $\nu<p_{2} \leq h_{X}$, we have

$$
\sum_{u \leq X, u: \text { prime }}\left(\frac{u}{p_{1}}\right)\left(\frac{u}{p_{2}}\right) \lambda(u) \ll X e^{-b \sqrt{\log X}},
$$

where $b$ is an absolute positive constant. From this and (4.13) we infer

$$
\begin{aligned}
\left|S_{2}\right| & \leq \sum_{\substack{p_{1}, p_{2}: \text { primes, } p_{1} \neq p_{2} \\
\nu<p_{1}, p_{2} \leq h_{X}}} \frac{1}{p_{1}^{\sigma_{1}} p_{2}^{\sigma_{1}}}\left|\sum_{\substack{u \leq X, u: \operatorname{prime} \\
u=\delta c \bmod Q}} \chi_{\delta u}\left(p_{1}\right) \overline{\chi_{\delta u}\left(p_{2}\right)}\right| \\
& =\sum_{\substack{p_{1}, p_{2}: \text { primes, } p_{1} \neq p_{2} \\
\nu<p_{1}, p_{2} \leq h_{X}}} \frac{1}{p_{1}^{\sigma_{1}} p_{2}^{\sigma_{1}}} O\left(X e^{-b \sqrt{\log X}}\right) \\
& \ll\left(\sum_{p \leq h_{X}} \frac{1}{p^{\sigma_{1}}}\right)^{2} O\left(X e^{-b \sqrt{\log X}}\right) \ll h_{X}^{2} X e^{-b \sqrt{\log X}} \\
& =o\left(\frac{X}{\log X}\right) .
\end{aligned}
$$

Consequently, for fixed $\nu$ we find, from (4.11), (4.12) and (4.15), that for all large $X$ and uniformly for $s \in \mathbb{C}$ with $\operatorname{Re} s \geq \sigma_{1}$,

$$
\sum_{\substack{u \leq X, u: \operatorname{prime} \\ u \equiv \delta c \bmod Q}}\left|\sum_{\nu<p \leq h_{X}} \frac{\chi_{\delta u}(p)}{p^{s}}\right|^{2} \ll \frac{\nu^{1-2 \sigma_{1}}}{2 \sigma_{1}-1} \frac{1}{\varphi(Q)} \frac{X}{\log X} .
$$


Note that the right-hand side of (4.16) is independent of $[c]_{Q} \in \mathcal{R}^{\gamma}$. Combining (4.16), (4.10), (4.3), (4.8) and (1.2), we conclude that

$$
\begin{aligned}
\sum_{q \in \mathcal{P}_{X, \nu}^{\gamma}(m, a)} \mid & \left.\sum_{\nu<p \leq h_{X}} \frac{\chi_{q}(p)}{p^{s}}\right|^{2} \ll \# \mathcal{R}^{\gamma} \frac{\nu^{1-2 \sigma_{1}}}{2 \sigma_{1}-1} \frac{1}{\varphi(Q)} \frac{X}{\log X} \\
& \ll \# \mathcal{R}^{\gamma} \frac{\nu^{1-2 \sigma_{1}}}{2 \sigma_{1}-1} \frac{\varphi(m)}{\varphi(Q)} \# \mathcal{P}_{X}^{\gamma}(m, a) \ll \frac{\nu^{1-2 \sigma_{1}}}{2 \sigma_{1}-1} C_{\nu}(m) \# \mathcal{P}_{X}^{\gamma}(m, a) .
\end{aligned}
$$

This completes the proof.

To obtain (4.14) we have used the Siegel-Walfisz theorem. We remark that actually, instead of the Siegel-Walfisz theorem, a weaker result (e.g. [Da, p. 123]) is sufficient since $p_{1} p_{2} Q \ll_{\nu} h_{X}^{2}=(\log \log X)^{4}$.

Proposition 4.3. Let $\sigma_{1}>1 / 2$ and $K$ be a compact subset of $\mathbb{C}$ such that $K \subset\left\{s \in \mathbb{C} \mid \operatorname{Re} s>\sigma_{1}\right\}$. Let $\varepsilon>0$. Then there exists a large real number $\nu_{0}=\nu_{0}\left(\sigma_{1}, K, \varepsilon, m\right)$ depending only on $\sigma_{1}, K, \varepsilon$ and $m$, and satisfying $\pi\left(\nu_{0}\right)>\pi(m)$ and the following. Fix any real number $\nu>\nu_{0}$. Let $a_{p} \in$ $\{1,-1\}$ for each prime $p$ satisfying $p \leq \nu$ and $p \nmid m$. Let $\mathcal{P}_{X, \nu}^{\gamma}(m, a), C_{\nu}(m)$ and $h_{X}$ be as in Lemma 4.2 for large $X$. Define $\mathcal{B}_{X, \nu}^{\gamma}(m, a)=\mathcal{B}_{X, \nu}^{\gamma}(m, a, \varepsilon$, $\left.\sigma_{1}, K,\left\{a_{p}\right\}\right)$ by

$$
\mathcal{B}_{X, \nu}^{\gamma}(m, a):=\left\{q \in \mathcal{P}_{X, \nu}^{\gamma}(m, a)\left|\max _{s \in K}\right| \sum_{\nu<p \leq h_{X}} \frac{\chi_{q}(p)}{p^{s}} \mid<\varepsilon\right\} .
$$

Then for all sufficiently large $X$ we have

$$
\frac{\# \mathcal{B}_{X, \nu}^{\gamma}(m, a)}{\# \mathcal{P}_{X}^{\gamma}(m, a)}>\frac{1}{2} C_{\nu}(m) .
$$

Proof. Set $\sigma_{2}=1+\sup \{\operatorname{Re} s \mid s \in K\}$ and $A=1+\sup \{|\operatorname{Im} s| \mid s \in K\}$. Let $U$ be the open rectangle $\left\{s \in \mathbb{C}\left|\sigma_{1}<\operatorname{Re} s<\sigma_{2},\right| \operatorname{Im} s \mid<A\right\}$ in $\mathbb{C}$, and then $U \supset K$. Take a large real number $\nu_{0}=\nu_{0}\left(\sigma_{1}, K, \varepsilon, m\right)$ satisfying $\pi\left(\nu_{0}\right)>\pi(m)$ and

$$
\left(\int_{U} 1 d \sigma d t\right) c \frac{\nu_{0}^{1-2 \sigma_{1}}}{2 \sigma_{1}-1}<\frac{\varepsilon^{2}}{4 b(U, K)^{2}}
$$

where $c$ is the absolute constant implied by the symbol $\ll$ in (4.9), and $b(U, K)$ is the constant in Lemma 2.2. Note that $\nu_{0}$ depends only on $\sigma_{1}, K, \varepsilon$ and $m$.

In the following we fix any $\nu>\nu_{0}$. For large $X$ we define

$$
\begin{aligned}
& \widetilde{\mathcal{B}}_{X, \nu}^{\gamma}(m, a) \\
& \quad:=\left\{\left.q \in \mathcal{P}_{X, \nu}^{\gamma}(m, a)\left|\int_{U}\right| \sum_{\nu<p \leq h_{X}} \frac{\chi_{q}(p)}{p^{s}}\right|^{2} d \sigma d t<\frac{\varepsilon^{2}}{b(U, K)^{2}}\right\} .
\end{aligned}
$$


By Lemma 2.2,

$$
\widetilde{\mathcal{B}}_{X, \nu}^{\gamma}(m, a) \subset \mathcal{B}_{X, \nu}^{\gamma}(m, a) .
$$

By Lemma 4.2 and (4.17), we have, for all large $X$,

$$
\begin{aligned}
\sum_{q \in \mathcal{P}_{X, \nu}^{\gamma}(m, a)} \int_{U} \mid \sum_{\nu<p \leq h_{X}} & \left.\frac{\chi_{q}(p)}{p^{s}}\right|^{2} d \sigma d t \\
& \leq\left(\int_{U} 1 d \sigma d t\right) c \frac{\nu^{1-2 \sigma_{1}}}{2 \sigma_{1}-1} C_{\nu}(m) \# \mathcal{P}_{X}^{\gamma}(m, a) \\
& <\frac{\varepsilon^{2}}{4 b(U, K)^{2}} C_{\nu}(m) \# \mathcal{P}_{X}^{\gamma}(m, a)
\end{aligned}
$$

Now we assume that there exists a large number $X$ such that

$$
\#\left(\mathcal{P}_{X, \nu}^{\gamma}(m, a)-\widetilde{\mathcal{B}}_{X, \nu}^{\gamma}(m, a)\right) \geq \frac{1}{4} C_{\nu}(m) \# \mathcal{P}_{X}^{\gamma}(m, a) .
$$

Then for this $X$ we have, using (4.18),

$$
\begin{aligned}
\sum_{q \in \mathcal{P}_{X, \nu}^{\gamma}(m, a)} \int_{U}\left|\sum_{\nu<p \leq h_{X}} \frac{\chi_{q}(p)}{p^{s}}\right|^{2} d \sigma d t & \\
& \geq \sum_{q \in \mathcal{P}_{X, \nu}^{\gamma}(m, a)-\widetilde{\mathcal{B}}_{X, \nu}^{\gamma}(m, a)} \int_{U}\left|\sum_{\nu<p \leq h_{X}} \frac{\chi_{q}(p)}{p^{s}}\right|^{2} d \sigma d t \\
& \geq \frac{1}{4} C_{\nu}(m) \# \mathcal{P}_{X}^{\gamma}(m, a) \frac{\varepsilon^{2}}{b(U, K)^{2}} .
\end{aligned}
$$

However, this contradicts (4.20). Hence for all large $X$ we have

$$
\#\left(\mathcal{P}_{X, \nu}^{\gamma}(m, a)-\widetilde{\mathcal{B}}_{X, \nu}^{\gamma}(m, a)\right)<\frac{1}{4} C_{\nu}(m) \# \mathcal{P}_{X}^{\gamma}(m, a)
$$

so

$$
\frac{\# \widetilde{\mathcal{B}}_{X, \nu}^{\gamma}(m, a)}{\# \mathcal{P}_{X}^{\gamma}(m, a)}>\frac{\# \mathcal{P}_{X, \nu}^{\gamma}(m, a)}{\# \mathcal{P}_{X}^{\gamma}(m, a)}-\frac{1}{4} C_{\nu}(m)
$$

Further, Lemma 4.1 implies that

$$
\frac{\# \mathcal{P}_{X, \nu}^{\gamma}(m, a)}{\# \mathcal{P}_{X}^{\gamma}(m, a)}>\frac{3}{4} C_{\nu}(m) \quad \text { if } X \text { is large enough. }
$$

Combining (4.19), (4.21) and (4.22), we conclude that if $X$ is large enough then

$$
\frac{\# \mathcal{B}_{X, \nu}^{\gamma}(m, a)}{\# \mathcal{P}_{X}^{\gamma}(m, a)} \geq \frac{\# \widetilde{\mathcal{B}}_{X, \nu}^{\gamma}(m, a)}{\# \mathcal{P}_{X}^{\gamma}(m, a)}>\frac{3}{4} C_{\nu}(m)-\frac{1}{4} C_{\nu}(m)=\frac{1}{2} C_{\nu}(m)
$$




\section{Proofs of Theorem 1.1 and its corollaries}

Proof of Theorem 1.1. Let $\varepsilon>0$ be an arbitrary small number. Take a real number $\sigma_{1}>1 / 2$ such that $K \subset\left\{s \in \mathbb{C} \mid \operatorname{Re} s>\sigma_{1}\right\}$. Fix a large positive number $\nu_{1}$ satisfying $\nu_{1}>\nu_{0}\left(\sigma_{1}, K, \varepsilon, m\right)$ and $\nu_{1}^{1-2 \sigma_{1}} /\left(2 \sigma_{1}-1\right)<\varepsilon$, where $\nu_{0}\left(\sigma_{1}, K, \varepsilon, m\right)$ is the constant in Proposition 4.3. We set $a_{2}$ to be 1 if $a \equiv 1$ or $7 \bmod 8$, and -1 if $a \equiv 3$ or $5 \bmod 8$. Further, we set $a_{p}=\left(\frac{\delta a}{p}\right)$ for each odd prime $p$ with $p \mid m$, where $\delta$ is as in (4.1).

As is shown in [MN1], there exists a holomorphic function $g(s)$ on $\Omega$ such that $g(x) \in \mathbb{R}$ for any $x \in \Omega \cap \mathbb{R}$ and

$$
h(s)=e^{g(s)} .
$$

Now Proposition 2.3 implies that there exist $\nu>\nu_{1}$ and $a_{p} \in\{1,-1\}$, for each prime $p$ with $p \leq \nu$ and $p \nmid m$, such that

$$
\max _{s \in K}\left|g(s)-\log \prod_{p \leq \nu}\left(1-\frac{a_{p}}{p^{s}}\right)^{-1}\right|<\varepsilon .
$$

For those $a_{p}$ 's, where $p \leq \nu$ and $p \nmid m$, we apply Proposition 4.3. Then for the above number $\nu$ and all large $X$, we have

$$
\frac{\# \mathcal{B}_{X, \nu}^{\gamma}(m, a)}{\# \mathcal{P}_{X}^{\gamma}(m, a)}>\frac{1}{2} C_{\nu}(m)
$$

Since $8 \mid m$, we have $q \equiv \delta a \bmod 8$ and $q \equiv \delta a \bmod p$ for $q \in \mathcal{P}^{\gamma}(m, a)$ and a prime $p$ with $p \mid m$. This and the definition of Kronecker's symbol yield $\chi_{q}(2)=a_{2}$ and $\chi_{q}(p)=\left(\frac{q}{p}\right)=\left(\frac{\delta a}{p}\right)=a_{p}$ for $q \in \mathcal{P}^{\gamma}(m, a)$ and an odd prime $p$ with $p \mid m$. Hence, from the definition of $\mathcal{B}_{X, \nu}^{\gamma}(m, a)$ we find that for every $q \in \mathcal{B}_{X, \nu}^{\gamma}(m, a)$ and all large $X$,

$$
\begin{aligned}
\max _{s \in K}\left|\log \prod_{p \leq \nu}\left(1-\frac{a_{p}}{p^{s}}\right)^{-1}-\log \prod_{p \leq h_{X}}\left(1-\frac{\chi_{q}(p)}{p^{s}}\right)^{-1}\right| \\
=\max _{s \in K}\left|\sum_{\nu<p \leq h_{X}} \frac{\chi_{q}(p)}{p^{s}}+\sum_{\nu<p \leq h_{X}} \sum_{n=2}^{\infty} \frac{\chi_{q}(p)^{n}}{n p^{n s}}\right| \\
\leq \max _{s \in K}\left|\sum_{\nu<p \leq h_{X}} \frac{\chi_{q}(p)}{p^{s}}\right|+\max _{s \in K}\left|\sum_{\nu<p \leq h_{X}} \sum_{n=2}^{\infty} \frac{\chi_{q}(p)^{n}}{n p^{n s}}\right| \\
\leq \varepsilon+O(\varepsilon) \ll \varepsilon,
\end{aligned}
$$

since

$$
\left|\sum_{\nu<p \leq h_{X}} \sum_{n=2}^{\infty} \frac{1}{n p^{n s}}\right| \ll \sum_{\nu<p \leq h_{X}} \frac{1}{p^{2 \sigma_{1}}} \ll \frac{\nu^{1-2 \sigma_{1}}}{2 \sigma_{1}-1}<\frac{\nu_{1}^{1-2 \sigma_{1}}}{2 \sigma_{1}-1}<\varepsilon .
$$


From (5.4) and (5.2) we deduce, for every $q \in \mathcal{B}_{X, \nu}^{\gamma}(m, a)$,

$$
\max _{s \in K}\left|g(s)-\log \prod_{p \leq h_{X}}\left(1-\frac{\chi_{q}(p)}{p^{s}}\right)^{-1}\right| \ll \varepsilon
$$

and therefore

$$
\begin{aligned}
\max _{s \in K}\left|\prod_{p \leq h_{X}}\left(1-\frac{\chi_{q}(p)}{p^{s}}\right)^{-1}-h(s)\right| \\
\quad=\max _{s \in K}\left|h(s)\left(\frac{\prod_{p \leq h_{X}}\left(1-\chi_{q}(p) / p^{s}\right)^{-1}}{h(s)}-1\right)\right| \\
\leq \max _{s \in K}|h(s)| \max _{s \in K}\left|e^{\log \prod_{p \leq h_{X}}\left(1-\chi_{q}(p) / p^{s}\right)^{-1}-g(s)}-1\right| \\
\ll_{K, h(s)} \varepsilon,
\end{aligned}
$$

using (5.1) and the fact that $e^{z}-1 \ll|z|$ if $|z|$ is small.

Let $\varepsilon_{1}$ be a small positive number such that

$$
\varepsilon_{1}<\min \left\{\varepsilon, \frac{C_{\nu}(m)}{2}\right\} \text {. }
$$

According to Proposition 3.2, if we put

$$
\begin{aligned}
& \mathcal{A}_{X}^{\gamma}(m, a) \\
& :=\left\{q \in \mathcal{P}_{X}^{\gamma}(m, a)\left|\max _{s \in K}\right| L\left(s, \chi_{q}\right)-\prod_{p \leq h_{X}}\left(1-\frac{\chi_{q}(p)}{p^{s}}\right)^{-1} \mid<\varepsilon_{1}\right\},
\end{aligned}
$$

then for all large $X$,

$$
\frac{\# \mathcal{A}_{X}^{\gamma}(m, a)}{\# \mathcal{P}_{X}^{\gamma}(m, a)}>1-\varepsilon_{1}
$$

By (5.6) and (5.5), every $q \in \mathcal{A}_{X}^{\gamma}(m, a) \cap \mathcal{B}_{X, \nu}^{\gamma}(m, a)$ satisfies

$$
\max _{s \in K}\left|L\left(s, \chi_{q}\right)-h(s)\right| \ll_{K, h(s)} \varepsilon .
$$

Furthermore, from (5.3) and (5.7) it follows that for the above number $\nu$ and all large $X$,

$$
\begin{aligned}
\#\left(\mathcal{A}_{X}^{\gamma}(m, a) \cap \mathcal{B}_{X, \nu}^{\gamma}(\right. & m, a)) \\
& \geq \# \mathcal{A}_{X}^{\gamma}(m, a)+\# \mathcal{B}_{X, \nu}^{\gamma}(m, a)-\# \mathcal{P}_{X}^{\gamma}(m, a) \\
& \geq\left(\frac{C_{\nu}(m)}{2}-\varepsilon_{1}\right) \# \mathcal{P}_{X}^{\gamma}(m, a) .
\end{aligned}
$$

Since $C_{\nu}(m) / 2-\varepsilon_{1}>0,(5.8)$ and (5.9) yield (1.3). This completes the proof. 
From Theorem 1.1 we can prove Corollaries $1.2-1.4$ by the same arguments as in the proofs of Corollaries 1.2-1.4 in [MN1], respectively.

6. On the line $\operatorname{Re} s=1$. In this section we prove Theorem 1.5. The next lemma is proved in [MN1].

Lemma 6.1. Let $t \in \mathbb{R}^{+}$and $y \in \mathbb{R}^{+}$be fixed. Then for any $z_{0} \in \mathbb{C}$ and $\varepsilon>0$, there exist $\nu \geq y$ and $c_{p} \in\{1,-1\}$, for each prime $p$ with $y \leq p \leq \nu$, such that

$$
\left|z_{0}-\sum_{y \leq p \leq \nu} \frac{c_{p}}{p^{1+i t}}\right|<\varepsilon .
$$

Proposition 6.2. Let $t \in \mathbb{R}^{+}$be fixed. Let $z \in \mathbb{C}$ and $\nu_{1} \in \mathbb{R}^{+}$with $\nu_{1}>m+1$. Let $a_{p} \in\{1,-1\}$ for each prime $p$ with $p \mid m$. Then for any $\varepsilon>0$ there exist $\nu>\nu_{1}$ and $a_{p} \in\{1,-1\}$, for each prime $p$ with $p \leq \nu$ and $p \nmid m$, such that

$$
\left|z-\log \prod_{p \leq \nu}\left(1-\frac{a_{p}}{p^{1+i t}}\right)^{-1}\right|<\varepsilon,
$$

where

$$
\begin{aligned}
\log \prod_{p \leq \nu}\left(1-\frac{a_{p}}{p^{1+i t}}\right)^{-1} & =-\sum_{p \leq \nu} \log \left(1-\frac{a_{p}}{p^{1+i t}}\right) \\
& =\sum_{p \leq \nu} \sum_{n=1}^{\infty} \frac{a_{p}^{n}}{n p^{n(1+i t)}} .
\end{aligned}
$$

Proof. The proof is similar to that of Proposition 2.3. Let $\varepsilon>0$ be arbitrary. Take a large number $y>\nu_{1}$ such that $1 / y<\varepsilon$. Then

$$
\sum_{p \geq y} \sum_{n=2}^{\infty} \frac{1}{n p^{n}} \ll \sum_{p \geq y} \frac{1}{p^{2}} \ll \frac{1}{y}<\varepsilon .
$$

Set $a_{p}=1$ for each prime $p$ with $p<y$ and $p \nmid m$.

From Lemma 6.1 it follows that there exist $\nu \geq y$ and $c_{p} \in\{1,-1\}$, for each prime $p$ with $y \leq p \leq \nu$, such that

$$
\left|\left(z-\sum_{p<y} \sum_{n=1}^{\infty} \frac{a_{p}^{n}}{n p^{n(1+i t)}}\right)-\sum_{y \leq p \leq \nu} \frac{c_{p}}{p^{1+i t}}\right|<\varepsilon .
$$

For each prime $p$ with $y \leq p \leq \nu$ we set $a_{p}=c_{p}$. Then we obtain, by (6.1) and (6.2), 


$$
\begin{aligned}
\left|z-\log \prod_{p \leq \nu}\left(1-\frac{a_{p}}{p^{1+i t}}\right)^{-1}\right| \\
=\left|z-\sum_{p<y} \sum_{n=1}^{\infty} \frac{a_{p}^{n}}{n p^{n(1+i t)}}-\sum_{y \leq p \leq \nu} \frac{c_{p}}{p^{1+i t}}-\sum_{y \leq p \leq \nu} \sum_{n=2}^{\infty} \frac{c_{p}^{n}}{n p^{n(1+i t)}}\right| \\
\leq\left|z-\sum_{p<y} \sum_{n=1}^{\infty} \frac{a_{p}^{n}}{n p^{n(1+i t)}}-\sum_{y \leq p \leq \nu} \frac{c_{p}}{p^{1+i t}}\right|+\left|\sum_{y \leq p \leq \nu} \sum_{n=2}^{\infty} \frac{c_{p}^{n}}{n p^{n(1+i t)}}\right| \\
<\varepsilon+\sum_{p \geq y} \sum_{n=2}^{\infty} \frac{1}{n p^{n}} \ll \varepsilon,
\end{aligned}
$$

which completes the proof.

Proof of Theorem 1.5. The proof is similar to that of Theorem 1.1 in Section 5. Since $L\left(1+i t, \chi_{q}\right)=\overline{L\left(1-i t, \chi_{q}\right)}$, it suffices to verify the assertion in the case $t>0$. Moreover, it suffices to consider the case $z_{0} \in \mathbb{C}-\{0\}$, since the set $\mathbb{C}-\{0\}$ is dense in $\mathbb{C}$.

Fix $z_{0} \in \mathbb{C}-\{0\}$ and $t>0$. Take a complex number $z$ such that $z_{0}=e^{z}$. Let $\varepsilon>0$ be an arbitrary small number. Take $\sigma_{1} \in \mathbb{R}$ with $1 / 2<\sigma_{1}<1$, and set $K=\{1+i t\}$. Take $\nu_{1} \in \mathbb{R}^{+}$so large that $1 / \nu_{1}<\varepsilon$ and $\nu_{1}>$ $\nu_{0}\left(\sigma_{1}, K, \varepsilon, m\right)$, where $\nu_{0}\left(\sigma_{1}, K, \varepsilon, m\right)$ is the constant in Proposition 4.3. We set $a_{2}$ to be 1 if $a \equiv 1$ or $7 \bmod 8$, and -1 if $a \equiv 3$ or $5 \bmod 8$. Further, we set $a_{p}=\left(\frac{\delta a}{p}\right)$ for each odd prime $p$ with $p \mid m$. According to Proposition 6.2, there exist $\nu>\nu_{1}$ and $a_{p} \in\{1,-1\}$, for each prime $p$ with $p \leq \nu$ and $p \nmid m$, such that

$$
\left|z-\log \prod_{p \leq \nu}\left(1-\frac{a_{p}}{p^{1+i t}}\right)^{-1}\right|<\varepsilon .
$$

For those $a_{p}$ 's, where $p \leq \nu$ and $p \nmid m$, we apply Proposition 4.3. Then for the above number $\nu$ and all large $X$, we have

$$
\frac{\# \mathcal{B}_{X, \nu}^{\gamma}(m, a)}{\# \mathcal{P}_{X}^{\gamma}(m, a)}>\frac{1}{2} C_{\nu}(m) \text {. }
$$

Noting $\chi_{q}(2)=a_{2}$ and $\chi_{q}(p)=a_{p}$ for $q \in \mathcal{P}^{\gamma}(m, a)$ and an odd prime $p$ with $p \mid m$, we have, for every $q \in \mathcal{B}_{X, \nu}^{\gamma}(m, a)$ and all large $X$,

$$
\begin{aligned}
\mid \log \prod_{p \leq \nu} & \left(1-\frac{a_{p}}{p^{1+i t}}\right)^{-1}-\log \prod_{p \leq h_{X}}\left(1-\frac{\chi_{q}(p)}{p^{1+i t}}\right)^{-1} \mid \\
& =\left|\sum_{\nu<p \leq h_{X}} \frac{\chi_{q}(p)}{p^{1+i t}}+\sum_{\nu<p \leq h_{X}} \sum_{n=2}^{\infty} \frac{\chi_{q}(p)^{n}}{n p^{n(1+i t)}}\right| \leq \varepsilon+O(\varepsilon) \ll \varepsilon,
\end{aligned}
$$


since

$$
\sum_{\nu<p \leq h_{X}} \sum_{n=2}^{\infty} \frac{1}{n p^{n}} \ll \sum_{\nu<p \leq h_{X}} \frac{1}{p^{2}} \ll \nu^{-1}<\nu_{1}^{-1}<\varepsilon .
$$

By (6.3) and (6.5), every $q \in \mathcal{B}_{X, \nu}^{\gamma}(m, a)$ satisfies

$$
\left|z-\log \prod_{p \leq h_{X}}\left(1-\frac{\chi_{q}(p)}{p^{1+i t}}\right)^{-1}\right| \ll \varepsilon
$$

and hence

$$
\begin{aligned}
\left|\prod_{p \leq h_{X}}\left(1-\frac{\chi_{q}(p)}{p^{1+i t}}\right)^{-1}-z_{0}\right| & =\left|z_{0}\left(\frac{\prod_{p \leq h_{X}}\left(1-\chi_{q}(p) / p^{1+i t}\right)^{-1}}{z_{0}}-1\right)\right| \\
& =\left|z_{0}\right|\left|e^{\log \prod_{p \leq h_{X}}\left(1-\chi_{q}(p) / p^{1+i t}\right)^{-1}-z}-1\right| \\
& \ll_{z_{0}} \varepsilon .
\end{aligned}
$$

Let $\varepsilon_{1}$ be a small positive number such that $\varepsilon_{1}<\min \left\{\varepsilon, C_{\nu}(m) / 2\right\}$. Proposition 3.2 implies that if we put

$$
\begin{aligned}
& \mathcal{A}_{X}^{\gamma}(m, a) \\
& :=\left\{q \in \mathcal{P}_{X}^{\gamma}(m, a)|| L\left(1+i t, \chi_{q}\right)-\prod_{p \leq h_{X}}\left(1-\frac{\chi_{q}(p)}{p^{1+i t}}\right)^{-1} \mid<\varepsilon_{1}\right\}
\end{aligned}
$$

then

$$
\frac{\# \mathcal{A}_{X}^{\gamma}(m, a)}{\# \mathcal{P}_{X}^{\gamma}(m, a)}>1-\varepsilon_{1}
$$

for all large $X$. Hence by (6.6) and (6.7) we conclude that every $q \in$ $\mathcal{A}_{X}^{\gamma}(m, a) \cap \mathcal{B}_{X, \nu}^{\gamma}(m, a)$ satisfies

$$
\left|L\left(1+i t, \chi_{q}\right)-z_{0}\right| \ll_{z_{0}} \varepsilon
$$

Furthermore, from (6.4) and (6.8) we see that for the above number $\nu$ and all $X$ sufficiently large,

$$
\#\left(\mathcal{A}_{X}^{\gamma}(m, a) \cap \mathcal{B}_{X, \nu}^{\gamma}(m, a)\right) \geq\left(\frac{C_{\nu}(m)}{2}-\varepsilon_{1}\right) \# \mathcal{P}_{X}^{\gamma}(m, a) .
$$

Since $C_{\nu}(m) / 2-\varepsilon_{1}>0,(6.9)$ and (6.10) complete the proof.

Acknowledgments. The authors would like to thank the referee for kind comments.

\section{References}

[Ay] R. Ayoub, An Introduction to the Analytic Theory of Numbers, Amer. Math. Soc., 1974 . 
[B1] B. Bagchi, The statistical behaviour and universality properties of the Riemann zeta-function and other allied Dirichlet series, Ph.D. thesis, Indian Statistical Institute, Calcutta, 1981.

[B2] -, A joint universality theorem for Dirichlet L-functions, Math. Z. 181 (1982), 319-334.

[BC] H. Bohr und R. Courant, Neue Anwendungen der Theorie der Diophantischen Approximationen auf die Riemannsche Zetafunktion, J. Reine Angew. Math. 144 (1914), 249-274.

[Da] H. Davenport, Multiplicative Number Theory, 3rd ed., Springer, 2000.

[El] P. D. T. A. Elliott, On the distribution of the values of quadratic L-series in the half-plane $\sigma>\frac{1}{2}$, Invent. Math. 21 (1973), 319-338.

[Em] K. M. Eminyan, $\chi$-universality of the Dirichlet L-function, Mat. Zametki 47 (1990), 132-137 (in Russian); English transl.: Math. Notes 47 (1990), 618-622.

[Go] S. M. Gonek, Analytic properties of zeta and L-functions, Ph.D. thesis, University of Michigan, 1979.

[KV] A. A. Karatsuba and S. M. Voronin, The Riemann Zeta-Function, de Gruyter, 1992.

[MN1] H. Mishou and H. Nagoshi, Functional distribution of $L\left(s, \chi_{d}\right)$ with real characters and denseness of quadratic class numbers, Trans. Amer. Math. Soc., to appear.

[MN2] -, - Character sums and class numbers of quadratic fields with prime discriminants, submitted.

[Ti] E. C. Titchmarsh, The Theory of the Riemann Zeta-Function, 2nd ed., Oxford Univ. Press, 1986.

[Vo] S. M. Voronin, Theorem on the "universality" of the Riemann zeta-function, Izv. Akad. Nauk SSSR Ser. Mat. 39 (1975), 475-486 (in Russian); English transl.: Math. USSR Izv. 9 (1975), 443-453.

Graduate School of Mathematics

Nagoya University

Chikusa-ku, Nagoya 464-8602, Japan

E-mail: m98018a@math.nagoya-u.ac.jp
Department of Mathematics

Faculty of Science

Niigata University

Niigata 950-2181, Japan

E-mail: nagoshih@ybb.ne.jp

Received on 7.3.2005

and in revised form on 20.3.2006 